\title{
O MODELO DE ECONOMIA CIRCULAR NO CONTEXTO DA PEQUENA EMPRESA: IDENTIFICAÇÃO DOS TERMOS E TENDÊNCIAS
}

Ludmila Neves Turchiari ${ }^{1}$

Ana Claudia Fernandes Terence ${ }^{1}$

Adriano Dos Reis Lucente ${ }^{1}$

Kelly Patricia Martins ${ }^{1}$

${ }^{1}$ Faculdade de Ciências Agrárias e Veterinárias / Universidade Estadual Paulista "Júlio de Mesquita Filho" 


\section{O MODELO DE ECONOMIA CIRCULAR NO CONTEXTO DA PEQUENA EMPRESA: IDENTIFICAÇÃO DOS TERMOS E TENDÊNCIAS}

\section{Resumo:}

Esse trabalho visa identificar os termos e as tendências em estudos de Economia Circular (EC) no contexto de pequenas empresas. Para tanto, realizou-se uma pesquisa bibliométrica nas bases Scopus e Web of Science com a consolidação dos dados na plataforma Citispace. A EC estabelece um modelo produtivo voltado ao aumento da vida útil de materiais e resíduos, contribuindo com a eliminação do uso de matérias-primas virgens. Quando tratada concomitante aos processos das pequenas empresas cria-se uma possibilidade de abordagem, pois estas, mesmo conscientes de seus impactos ambientais, deparam-se com custos de implantação, remanufatura e competências técnicas. Estudos sobre EC e pequenas empresas vêm crescendo nos últimos anos - há um aumento de publicações a partir de 2018 , denotando a sua atualidade. Este estudo pretende contribuir com a identificação dos principais autores, periódicos e publicações, bem como palavras-chave relacionadas ao tema, como simbiose industrial e economia verde, apontando informações relevantes aos pesquisadores.

Palavras-chave: Economia Circular; Simbiose industrial; Economia Verde; Bibliometria.

\section{INTRODUÇÃO}

O consumo global de matérias-primas é um agravante no cenário atual, o que torna necessária a definição e o estabelecimento de estratégias para reduzir a quantidade e a dependência da extração e importação de matérias-primas, melhorando, consequentemente, a sustentabilidade das empresas. Neste contexto, é essencial promover estratégias de Economia Circular (EC), como parcerias de simbiose industrial, na qual as empresas utilizam os resíduos de outras indústrias como matéria-prima (PATRICIO et al., 2018).

A EC é considerada uma iniciativa sustentável relevante para a minimização de resíduos em empresas de manufatura, mas há poucas pesquisas disponíveis sobre a prontidão da EC em micro, pequenas e médias empresas (SINGH; CHAKRABORTY; ROY, 2018).

A justificativa para a realização deste estudo se dá por meio do enfoque dos temas de EC concomitante ao de pequena empresa, pois ambos são considerados relativamente recentes e de grande importância para a sociedade em geral. Portanto, credita-se a necessidade de análise e revisão do panorama intelectual e de tendências emergentes na literatura científica de tais temas.

Considerando-se a relevância atual da abordagem do tema EC na pequena empresa, este trabalho apresenta um estudo bibliométrico que tem por objetivo identificar termos que envolvam o tema e, assim, identificar tendências e informações relevantes à compreensão e aprofundamento do assunto. 


\section{REFERENCIAL TEÓRICO}

\subsection{ECONOMIA CIRCULAR}

A EC é uma estratégia simples, contudo convincente, que visa reduzir tanto a entrada de materiais virgens e a produção de resíduos, fechando ciclos econômicos e ecológicos de fluxos de recursos (HAAS et al, 2015).

A pesquisadora Ellen McArthur, considerada uma referência em estudos de EC, em McArthur (2015), tornou-se uma das grandes difusoras do conceito pelo mundo através do seu Instituto Ellen McArthur de Economia Circular. Para iniciar a abordagem, a autora salienta o modelo tradicional econômico, que, por muitos anos não apresentou mudanças que o trouxessem ao patamar de um modelo sustentável de produção.

O modelo econômico linear de 'extrair, transformar, descartar' da atualidade está atingindo seus limites físicos e, segundo McArthur (2015), a EC é uma alternativa atraente que busca redefinir a noção de crescimento, com foco em benefícios para toda a sociedade. Isto envolve dissociar a atividade econômica do consumo de recursos finitos e eliminar resíduos do sistema por princípio. Apoiado por uma transição para fontes de energia renovável, o modelo circular constrói capital econômico, natural e social.

Assim, a EC é considerada um sistema econômico que se concentra em maximizar a reutilização de recursos e produtos e minimizar sua depreciação (BREURE; LIJZENA; MARINGC, 2018). O autor afirma que a EC influencia na depreciação e na condição do solo, sendo fator relevante de uma boa gestão. A gestão concisa dos recursos, segundo ele, determina o sucesso do processo.

A Figura 1 retrata o diagrama da Economia Linear, com a realização de reciclagem de materiais e da Economia Circular, pontuando a entrada de recursos no ciclo da economia em um sistema que alia produção e consumo por meio do uso sustentável de recursos em um ciclo fechado.

\section{Figura 1 - Diagrama da Economia Linear e da Economia Circular}

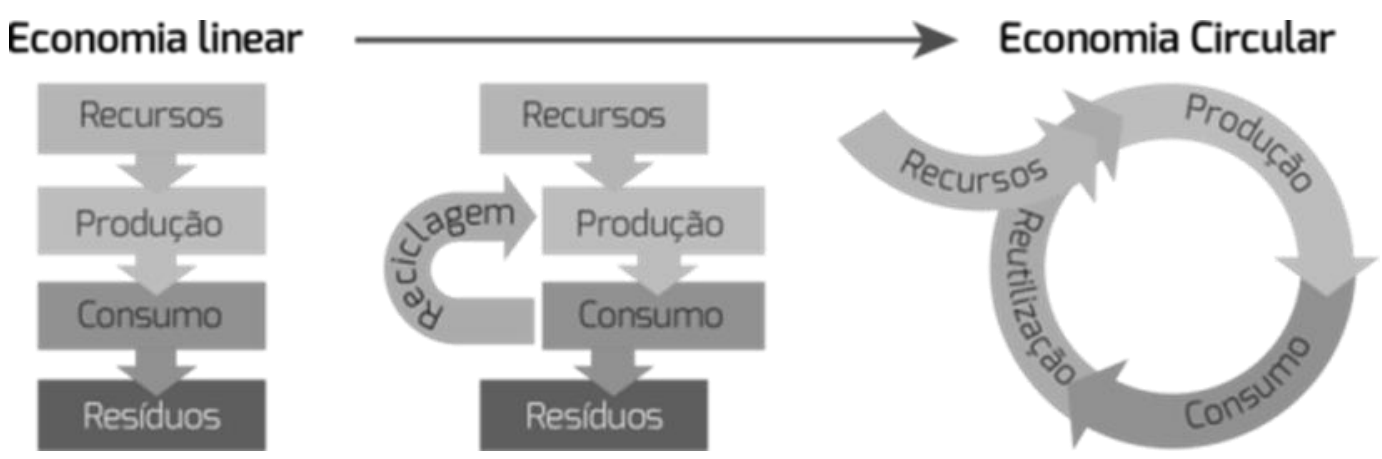

Fonte: Circular Economy (2019)

Vale considerar que, embora seja uma das ações consideradas sustentáveis em um processo produtivo, a reciclagem não pode ser considerada como prioridade na $\mathrm{EC}$, pois não sustenta o que envolve sua teoria. Essa perspectiva considera soluções menos sustentáveis, limitando a escolha de ações dos outros princípios (redução e reutilização) e criando tendência 
de desconsiderar ações de prevenção a exemplo do eco design que, por sua vez, sugere repensar e limitar recursos circulares (CIRCULAR ECONOMY, 2019; GHISELLINI; CIALANI; ULGIATI,2016)

A EC já é uma realidade e, em alguns países, se transformou em modelo normativo. Segundo Feng et al. (2012), a China possui a Lei do Desenvolvimento da Economia Circular, que compõe uma das diretrizes sustentáveis do país, com regras e regulamentos formados em resposta às políticas e leis nacionais, com a produção de efeitos significativos.

A implantação de ações, práticas e princípios de EC possui o potencial de gerar diminuição de custos, crescimento econômico, criação de empregos, inovação, produtividade, eficiência na utilização de recursos e conservação de recursos e do ecossistema (PAULIUK; WANG; MÜLLER, 2012). Embora seja benéfica ao meio ambiente é importante que seja integrada às políticas econômicas dos países de modo a não trazer prejuízo financeiro para quem implanta o modelo, para, assim, tornar-se uma teoria aceita e disseminada.

Em consonância a essa situação, Di Maio et al. (2017) revelam em seu estudo que produtos deixam de servir suas funções ao longo do seu ciclo e duração de vida, tornando-se cara a sua reintrodução ao processo. Portanto, cabe aqui aos formuladores de políticas a definição de ações que facilitem a implantação e a adequação ao modelo circular.

"Desenvolvimento sustentável só tem sentido se houver uma postura global e transversal e se implantado em todas as regiões do mundo". É assim que Laville (2009, p. 257) define sustentabilidade. Portanto, além das organizações serem consideradas sustentáveis grande parte do mundo terá de ser também.

Por isso, é eminente e urgente a análise em torno do tema de EC, pois considera e projeta a diminuição do impacto negativo causado pelo processo produtivo linear descrito. Esse tema torna-se relevante já que, além dos benefícios, criam expectativas de cenários futuros positivos na perspectiva da sustentabilidade.

\subsection{A PEQUENA EMPRESA NO MODELO CIRCULAR}

As pequenas empresas estão cada vez mais conscientes dos benefícios de seguir conceitos de EC e melhorar a eficiência dos recursos, como economizar custos e criar vantagens competitivas. Ao mesmo tempo, porém, várias barreiras colocam desafios às pequenas empresas na sua transição para uma EC, nomeadamente as faltas de recursos financeiros e de competências técnicas (RIZOS et al, 2016).

Daddi et al. (2019) citam que a EC cria desafios contrastantes para as empresas, o que leva a tensões paradoxais. Por um lado, as empresas buscam metas de EC como o uso de matérias-primas recicladas, destacando, assim, seu compromisso ambiental. Por outro lado, tal uso pode afetar a qualidade e, consequentemente, a competitividade dos produtos.

Além disso, Ormazabal et al (2018) revelam que os principais resultados mostram que as empresas estão focadas no cumprimento da lei e, em muitos casos, estão preocupadas com sua imagem. Além disso, eles não tendem a se comprometer com questões ambientais porque não acreditam que isso aumentaria seus lucros e competitividade.

A simbiose industrial também é um termo encontrado na literatura que trata da EC na pequena empresa, pois é um modelo que envolve inúmeras empresas que podem suprir uma única cadeia. A simbiose industrial, como um subcampo da ecologia industrial, diz respeito à 
cooperação entre empresas industriais na gestão de recursos, particularmente subprodutos, de forma que o desperdício de uma empresa se torna a entrada de outra (MULROW et al, 2017). Ambos os conceitos são tipicamente considerados em escalas que variam de parques industriais a cadeias de suprimento globais, mas, raramente na escala de uma única instalação que abriga várias empresas. Além disso, os conceitos de EC e simbiose industrial possuem requisitos e implicações que vão além do rastreamento e coordenação dos fluxos de materiais (MULROW et al, 2017).

\section{PROCEDIMENTOS METODOLÓGICOS}

O procedimento metodológico utilizado neste é teórico, de caráter descritivo e abordagem quali-quantitativa. Já o método de pesquisa utilizada foi a bibliometria. O tema de EC e pequenas empresas, por ser recente, requer maior apreciação e a bibliometria, nesse caso, oferece subsídios para estudos mais aprofundados fornecendo, também, identificação de possíveis lacunas (CRUZ; RIBEIRO, 2003), bem como um panorama geral da produção científica da área.

A busca de materiais foi realizada nas bases Scopus e Web Of Science, em função de sua representatividade e seu reconhecimento internacional. Para se atingir o objetivo proposto, este estudo foi realizado em quatro etapas: 1) definição de palavras-chave; 2) identificação, acesso e seleção de material nas bases de dados; 3) análise bibliométrica; 4) descrição dos termos identificados.

Para a busca de material foi estabelecido um horizonte temporal, considerando o período de 2000 a 2019, visando obter o maior tempo de cobertura possível do período em que as publicações do tema se iniciaram. Cabe ressaltar, também, que este é o período disponível para acesso e que o ano de 2019 apresenta um número reduzido de publicações, pois as publicações são referentes apenas aos dois primeiros meses desse ano.

A identificação do material foi realizada por meio da identificação de palavras-chave contidas nos abstracts de cada artigo. A definição deste critério está fundamentada na Lei Bibliométrica de Zipf que, conforme Macias-Chapula (1998) que permite estimar as frequências de ocorrência das palavras-chaves de um determinado texto científico. As palavraschave selecionadas são apresentadas na string a seguir:

STRING: (TITLE-ABS-KEY ( "circular economy" OR "circular business model" ) AND TITLE-ABS-KEY ( "craddle to craddle" OR "industrial symbiosis" OR "green economy" ) AND TITLE-ABS-KEY ( "small enterprise*" OR "sme*" OR "small compan*" OR "small firm*" OR " small medium enterprise*" )

Foram identificados 19 artigos e, após a adoção dos critérios de seleção, foram utilizados 15 artigos neste trabalho. A Figura 2 ilustra as etapas realizadas na coleta de dados que foram a identificação, acesso e seleção de material. 
Figura 2 - Etapas para a coleta de dados

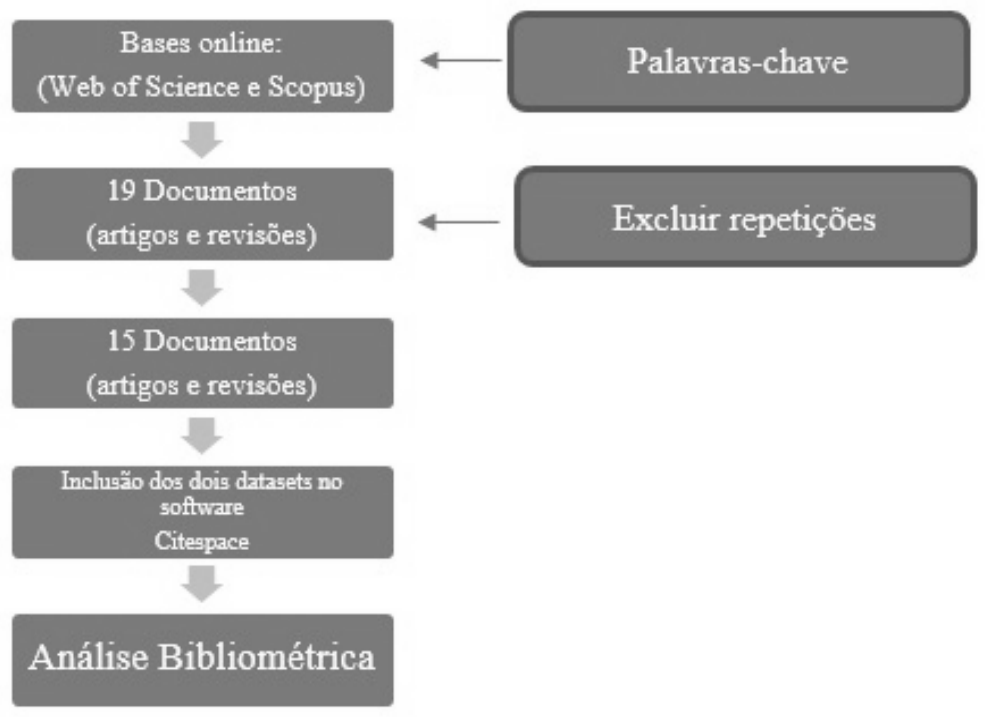

Fonte: Elaborado pelos autores

\subsection{MÉTODO DE ANÁLISE DE DADOS}

Para a realização da análise dos dados foram determinadas categorias, fundamentadas nos conceitos de bibliometria. As análises também foram conduzidas usando o aplicativo CiteSpace (Chen et al. 2010), pois tal aplicativo permitiu a condensação das duas bases científicas Web of Science e Scopus em uma única base de dados (dataset). O Quadro 1 sintetiza as categorias que serviram de base de dados para análise.

\section{Quadro 1 - Indicadores e variáveis para a análise bibliométrica}

\begin{tabular}{|l|l|}
\hline \multicolumn{1}{|c|}{ Indicadores } & \multicolumn{1}{c|}{ Variáveis } \\
\hline Principais artigos sobre o tema & Quantidade de citações e referências \\
\hline Publicações ao longo dos anos & Quantidade de publicações sobre o tema por ano \\
\hline Assuntos focos & $\begin{array}{l}\text { Identificação de assuntos comuns entre os artigos e sua } \\
\text { caracterização }\end{array}$ \\
\hline Palavras-chave & Quantidade de palavras-chave em comuns nesses artigos \\
\hline Redes de conexões & Palavras-chaves mais citadas em conjunto \\
\hline $\begin{array}{l}\text { Clusters de autores mais relevantes } \\
\text { e o ano da publicação }\end{array}$ & $\begin{array}{l}\text { Quantidade de artigos publicados x co-citações x autores } \\
\text { mais citados x ano de publicação }\end{array}$ \\
\hline Publicação por países & Identificação dos países e da quantidade de publicações \\
\hline Títulos da fonte & $\begin{array}{l}\text { Identificação das principais fontes e das quantidades de } \\
\text { publicações }\end{array}$ \\
\hline
\end{tabular}

Fonte: Elaborado pelos autores 


\section{APRESENTAÇÃO DOS RESULTADOS E DISCUSSÕES}

Buscou-se realizar um levantamento dos estudos sobre o tema EC e pequenas empresas de forma combinada, ou seja, a quantificação das publicações abordando os dois temas simultaneamente.

Para apresentação dos principais artigos o aplicativo Citespace possibilitou a compilação dos resultados das duas bases em apenas um referencial, facilitando a geração dos gráficos e análise do conteúdo. O Quadro 2 apresenta os principais artigos de acordo com os números de citações, dado gerado através do aplicativo Citespace.

Quadro 2 - Publicações sobre economia circular no contexto da pequena empresa

\begin{tabular}{|c|c|c|c|}
\hline Autor(a) & Título & Ano & Citações \\
\hline WEN;MENG & $\begin{array}{l}\text { Quantitative assessment of industrial symbiosis for the } \\
\text { promotion of circular economy: A case study of the printed } \\
\text { circuit boards industry in China's Suzhou New District }\end{array}$ & 2015 & 62 \\
\hline RIZOS et al. & $\begin{array}{l}\text { Implementation of circular economy business models by } \\
\text { small and medium-sized enterprises (SMEs): Barriers and } \\
\text { enablers }\end{array}$ & 2016 & 57 \\
\hline DADDI; NUCCI; IRALDO & $\begin{array}{l}\text { Using Life Cycle Assessment (LCA) to measure the } \\
\text { environmental benefits of industrial symbiosis in an } \\
\text { industrial cluster of SMEs }\end{array}$ & 2017 & 24 \\
\hline MULROW et al. & Industrial Symbiosis at the Facility Scale & 2017 & 18 \\
\hline ORMAZABAL et al & $\begin{array}{l}\text { Circular Economy in Spanish SMEs: Challenges and } \\
\text { opportunities }\end{array}$ & 2018 & 14 \\
\hline $\begin{array}{l}\text { RAZMINIENE; } \\
\text { TVARONAVICIENE }\end{array}$ & $\begin{array}{l}\text { Detecting the linkages between clusters and circular } \\
\text { economy }\end{array}$ & 2018 & 7 \\
\hline $\begin{array}{l}\text { SINGH; CHAKRABORTY; } \\
\text { ROY }\end{array}$ & $\begin{array}{l}\text { Developing an extended theory of planned behavior model } \\
\text { to explore circular economy readiness in manufacturing } \\
M S M E s, \text { India }\end{array}$ & 2018 & 6 \\
\hline SELLITTO; MURAKAMI & $\begin{array}{l}\text { Industrial symbiosis: A case study involving a steelmaking, } \\
\text { a cement manufacturing, and a zinc smelting plant }\end{array}$ & 2018 & 2 \\
\hline $\begin{array}{l}\text { TURJAK; UNUKIĆ; } \\
\text { LIOVIC }\end{array}$ & Aspects of the transition of SMEs in the Republic of Croatia & 2018 & 1 \\
\hline $\begin{array}{l}\text { PIGOSSO; } \\
\text { SCHMIEGELOW; } \\
\text { ANDERSEN }\end{array}$ & $\begin{array}{l}\text { Measuring the Readiness of SMEs for Eco-Innovation and } \\
\text { Industrial Symbiosis: Development of a Screening Tool }\end{array}$ & 2018 & 0 \\
\hline $\begin{array}{l}\text { PATRICIO; AXELSSON; } \\
\text { BLOME; ROSADO }\end{array}$ & $\begin{array}{l}\text { Enabling industrial symbiosis collaborations between } \\
\text { SMEs from a regional perspective }\end{array}$ & 2018 & 0 \\
\hline ÇOLAK; AKCENGIZ & $\begin{array}{l}\text { Transition from conventional to sustainable production: A } \\
\text { case study in OSTIM organized industrial zone }\end{array}$ & 2018 & 0 \\
\hline ALI; WANG; ALVARADO & $\begin{array}{l}\text { Facilitating industrial symbiosis to achieve circular } \\
\text { economy using value-added by design: A case study in } \\
\text { transforming the automobile industry sheet metal waste- } \\
\text { flow into Voronoi facade systems }\end{array}$ & 2019 & 0 \\
\hline
\end{tabular}




\begin{tabular}{|l|l|l|l|}
\hline Autor(a) & Título & Ano & Citações \\
\hline DADDI et al & $\begin{array}{l}\text { Paradoxical tensions and corporate sustainability: A focus } \\
\text { on circular economy business cases }\end{array}$ & 2019 & 0 \\
\hline ROMNÉE et al & $\begin{array}{l}\text { A greenhouse that reduces greenhouse effect: } \text { How to } \\
\text { create a circular activity with construction waste? }\end{array}$ & 2019 & 0 \\
\hline
\end{tabular}

Fonte: Elaborado pelos autores com o uso do Citespace

Sobre os assuntos abordados por esses quinze artigos, identificou-se uma categorização desses assuntos em duas bases comuns: 1) implantação de EC em pequenas empresas e; 2) simbiose industrial e eco parques industriais. Tais assuntos são de grande importância no contexto de EC, uma vez que, apesar do alto potencial de negócios, inovação e sustentabilidade ligado à eco-inovação que leva a grande tendência a simbiose industrial, em contrapartida, a implementação limitada pode ser observada em pequenas e médias empresas (PIGOSSO et al, 2018). Dentre os quinze artigos, sete $(46,6 \%)$ abordaram assuntos de implantação de EC em pequenas empresas e oito $(53,4 \%)$ abordaram assuntos de simbiose industrial e eco parques industriais.

De acordo com Wen e Meng (2015), só é possível a construção de um sistema de simbiose industrial se as cadeias produtivas forem alocadas em parques eco-industriais. Mulrow et al (2017), por sua vez, explicam que, além da situação de parques tecnológicos, entende-se simbiose como gestão de recursos, principalmente subprodutos, evitando desperdícios entre as empresas.

Tais subprodutos e insumos reciclados e seus aspectos ambientais até então eram ignorados pelas gestões convencionais de parques industriais. Por isso, a simbiose industrial trata-se de um mecanismo que considera fatores econômicos, ecológicos e energéticos frisando a reutilização de matérias primas e uso eficiente de recursos (ÇOLAK e AKCENGIZ, 2018).

Para a avaliação dos efeitos da simbiose industrial, Daddi, Nucci e Iraldo (2017) e Romnée et al (2019) defendem que, no sentido de aplicação de EC, é importante que se utilizem ferramentas de avaliação como análise do ciclo de vida, que consiste em uma avaliação das entradas e saídas e os impactos oriundos de um processo ao longo de toda a cadeia produtiva. Num estudo avaliativo de um produto ou serviço, todas as saídas de recursos e emissões para o meio ambiente, são avaliadas de forma quantitativa ao longo de todo o ciclo (FERREIRA, 2004).

Ressalte-se que quanto à abordagem da implantação de EC em pequenas empresas, identificam-se desafios contrastantes para essas empresas, o que leva a tensões paradoxais. Por um lado, as empresas buscam metas de EC, como o uso de matérias-primas recicladas, destacando, assim, seu compromisso ambiental. Por outro lado, tal uso pode afetar a qualidade e, consequentemente, a competitividade dos produtos (DADDY et al, 2019).

No entanto, é evidente, apesar de tais obstáculos, que existe uma parcela de empresas conscientes quanto aos benefícios em aumento de eficiência na implantação de EC. Desse modo, considera-se que há diferença nos resultados, quanto a economia de custos com materiais, vantagens competitivas e acessos a novos mercados (RIZOS et al, 2016; SINGH; CHAKRABORTY; ROY, 2018). 
Ormazabal et al (2018) citam que, quando se analisam as empresas em processo de implantação de EC, observa-se que elas entendem a importância do cumprimento da lei e a questão de sua imagem. No entanto, ainda não acreditam que o compromisso ambiental possibilita lucro e competitividade.

Não à toa, embora algumas empresas não entendam os benefícios quanto à implantação de EC, é indiscutível que o assunto esteja ganhando proporção e reconhecimento científico. Também se verificou que houve um aumento do número de publicações ao longo dos anos sobre o tema de EC e pequenas empresas (Gráfico 1), com destaque para o ano de 2018, com um crescimento de $300 \%$ em relação ao ano anterior. Ressalte-se que esta abordagem é considerada recente na literatura internacional apontando crescimento a partir de 2018.

\section{Gráfico 1 - Publicações ao longo dos anos}

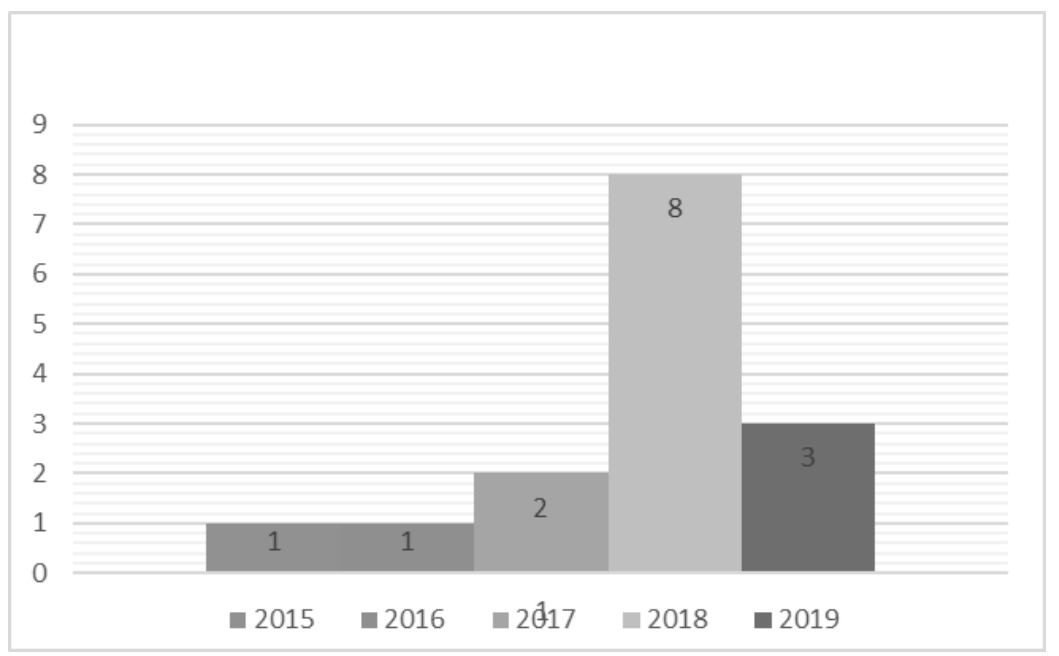

Fonte: Elaborado pelos autores

As palavras-chave identificadas nos artigos sobre o tema EC e pequenas empresas, mostradas no Gráfico 2, apresentam tendências de novos termos novos na área. Ao mesmo tempo em que palavras como gestão ambiental, economia verde e cadeia de suprimentos reforçam os envolvimentos dos aspectos industriais aos ambientais, o termo simbiose industrial revela ser o de maior força, nesse sentido, em sequência do termo EC.

Gráfico 2- Palavras-chave dos artigos sobre o tema EC e pequenas empresas 


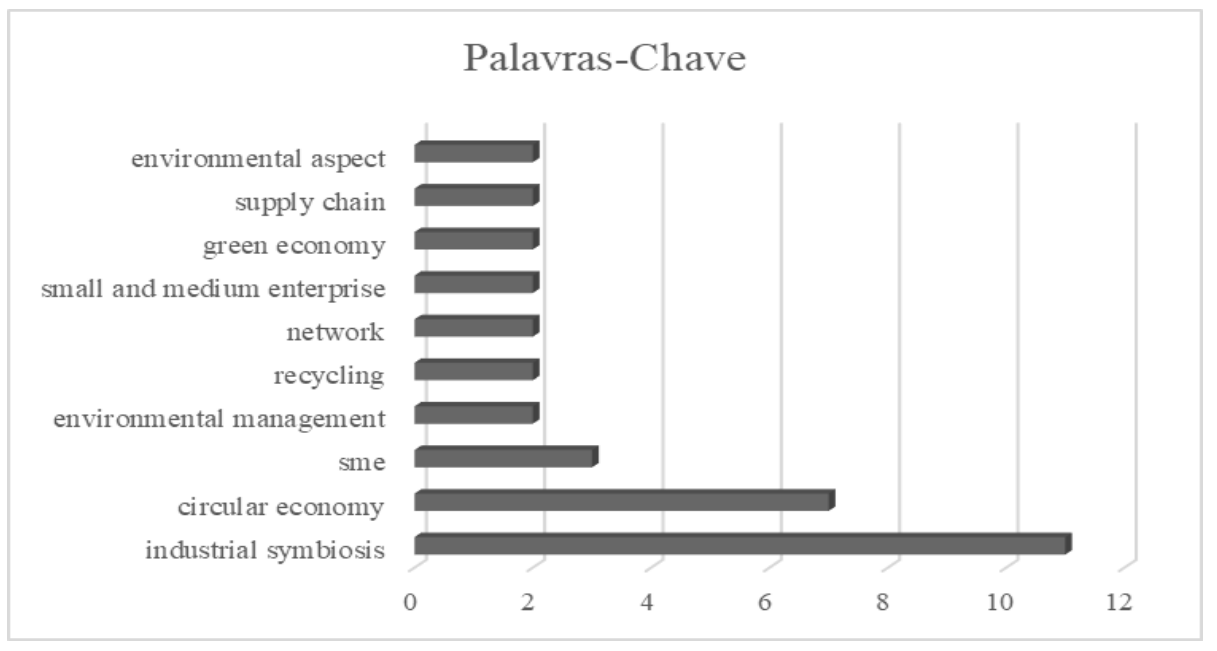

Fonte: Elaborado pelos autores

As redes de conexões entre as palavras-chaves mostram a conectividade entre os termos (Figura 3). É possível verificar que, embora haja engajamento de algumas palavras com outras, ainda existem poucas conexões entre as palavras "pequenas empresas" e "economia circular". SMEs, que seria o termo inglês para pequenas empresas, assim como "small and medium enterprises", possuem maiores conexões com termos mais tradicionais às indústrias como gestão ambiental. Enquanto isso, termos como Economia Circular e Simbiose Industrial possuem maiores forças e conexões quando ligados a termos como reciclagem, economia verde, aspectos ambientais e Gestão Ambiental.

\section{Figura 3 - Redes de Conexões das palavras-chaves}

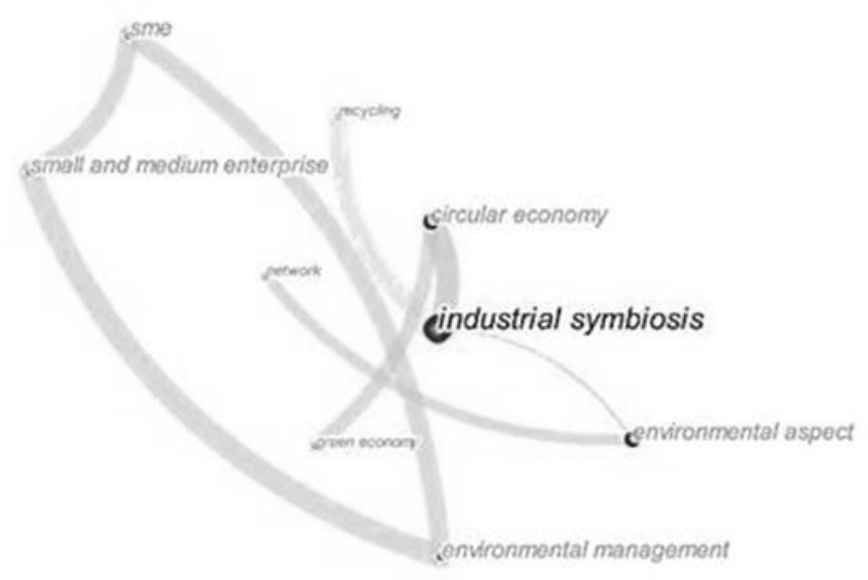

Fonte: Elaborado pelos autores

Um indicador útil à visão geral das publicações da área são os nós de clusters que compreendem um nó que possui altas pontuações de centralidade de intermediação (betweenness centrality scores) (CHEN et al. 2010). No estudo foi possível identificar 16 
clusters de autores relevantes utilizando variações como autores, citações e referências. Nesse caso, o tamanho do nó (cluster) determina sua importância.

Os clusters foram alinhados conforme as publicações ao longo dos anos para desenhar um cenário de importância dos autores e os anos relacionados. Na Figura 4 é possível analisar que, no que se refere ao tema de economia circular e pequena empresa, existem autores que possuem maiores referências na área como Geng Y, Cecelja F., Geissdoerfer M e Blomsma F., sendo, este último, o mais atual.

Figura 4 - Clusters de autores mais relevantes e o ano da publicação

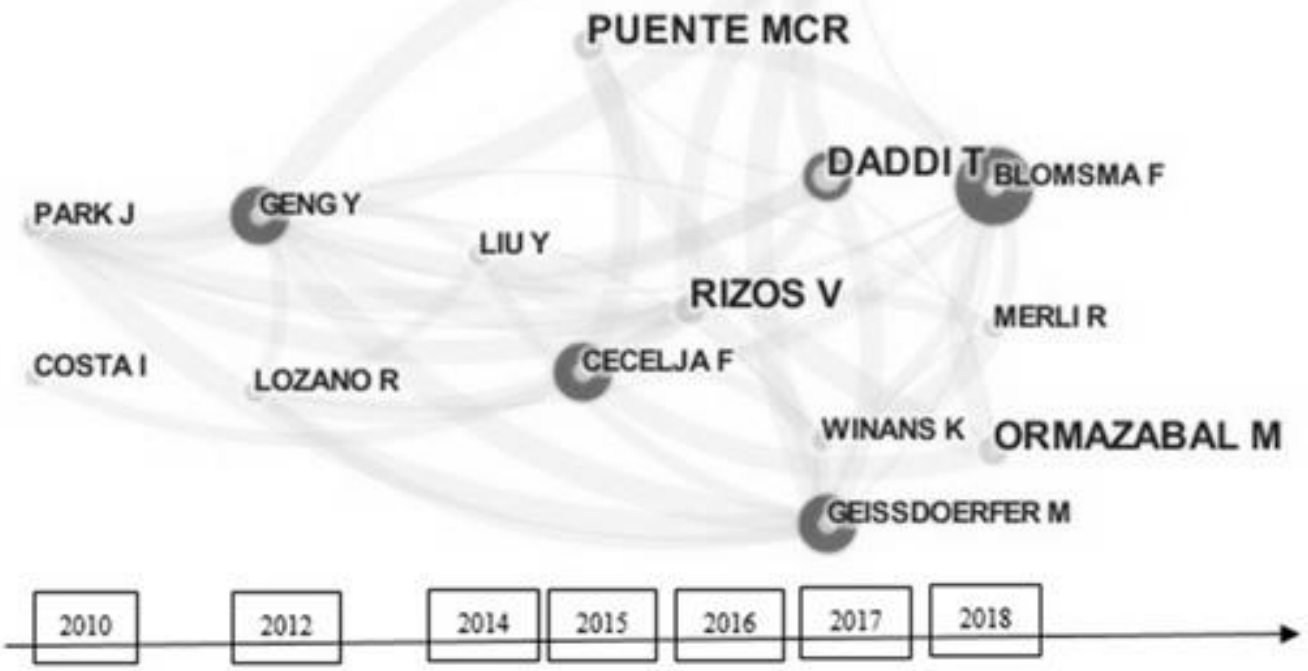

Fonte: Elaborado pelos autores

Como a seleção dos trabalhos resultou em apenas 15 artigos, verificou-se uma distribuição homogênea entre os países com publicação na temática, destacando-se dois países com maior número: Estados Unidos e Itália (Figura 5). Destacam-se, também, duas publicações do mesmo autor italiano Daddy T.

Vale considerar que, dentre todos os artigos, foi identificado um de origem brasileira. Embora seja um número reduzido, mostra que existem pesquisas em andamento desse potencial tema no país. 


\section{Figura 5 - Publicação por país}

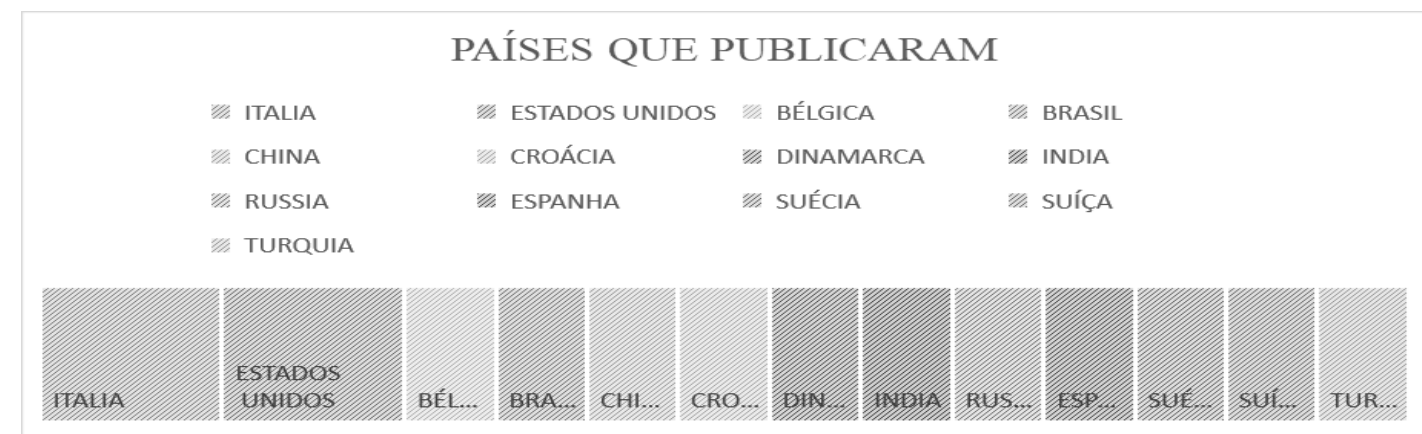

Fonte: Elaborado pelos autores

Das fontes que publicam os resultados das pesquisas sobre a temática ECe pequena empresa, é nítida a submissão das investigações para o Journal of Cleaner Production. As demais fontes foram distribuídas de forma homogênea e, como pode ser visualizado no gráfico 4, não houve nenhum outro destaque, mas é importante considerar-se todas as fontes, uma vez que a amostra deste estudo é pequena.

\section{Gráfico 4 - Periódicos das publicações}

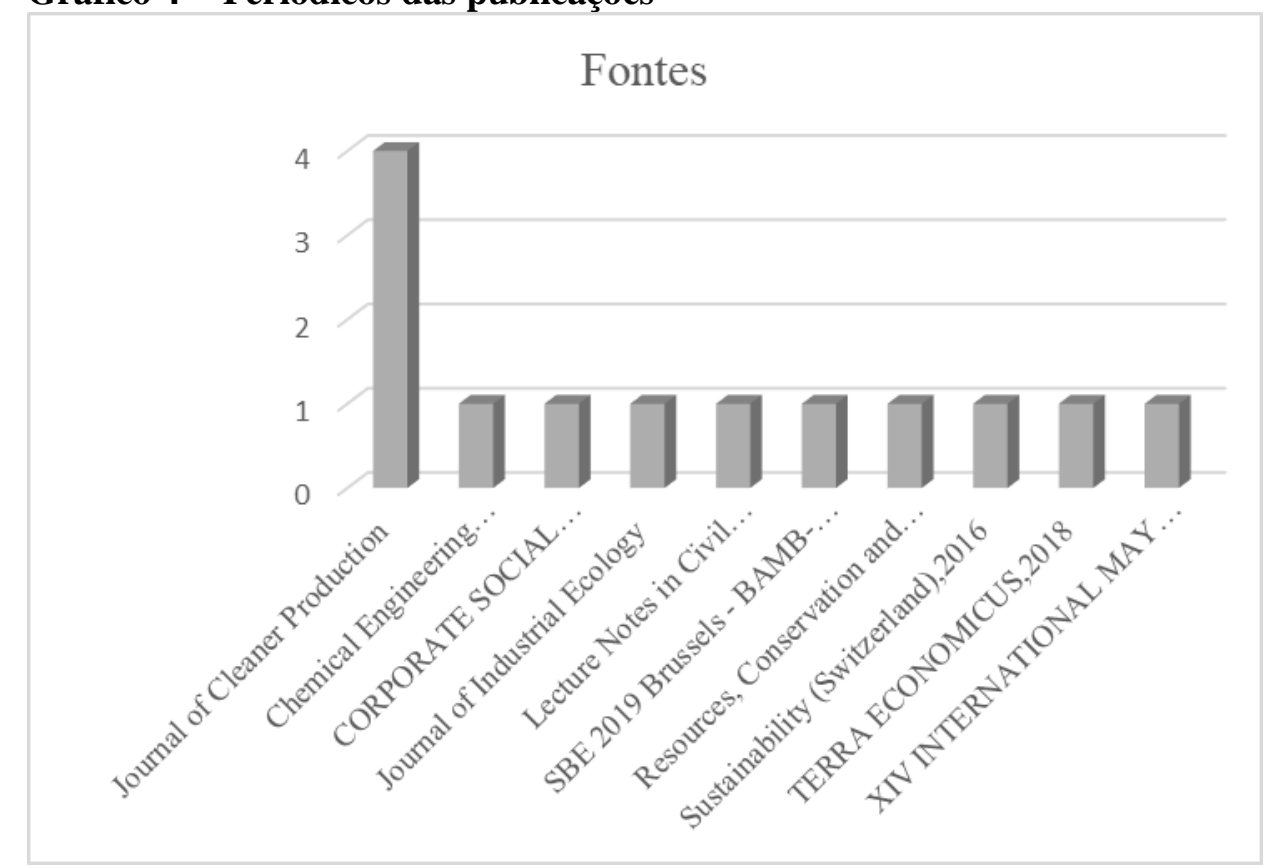

Fonte: Elaborado pelos autores

A bibliometria em conjunto com os demais levantamentos de dados e informações do referido trabalho possibilita compreender de forma ampla os principais estudos sobre o modelo da EC no contexto da pequena empresa. 


\section{CONSIDERAÇÕES FINAIS}

Este artigo teve o intuito de traçar um panorama geral das publicações na temática relacionada aos modelos de EC no âmbito da pequena empresa. Para tanto, a partir da análise de 15 artigos publicados no período 2000 a 2019, nas bases de dados Web of Science e Scopus, foram identificados autores, periódicos, países, redes de conexões de autores e periódicos relevantes.

O tema EC juntamente com pequena empresa teve um aumento significativo de publicações nos últimos anos, obtendo destaque os anos de 2017 e 2018 com um crescimento de $300 \%$, indicando a sua importância e o ganho de atenção no âmbito acadêmico.

Quanto às palavras-chaves, destaca-se a Simbiose Industrial como a mais citada seguida pelo próprio termo principal deste estudo que é a EC, apontando que os estudos envolvendo EC e pequenas empresas está diretamente ligado a transformações dos processos comuns em modelos de simbiose industrial.

Além disso, houve a apresentação de um reforço entre termos como Gestão Ambiental, Economia Verde e Cadeia de Suprimentos, bem como um potencial crescimento em conexões dos termos EC e Simbiose Industrial com reciclagem, economia verde, aspectos ambientais e gestão ambiental, ressaltando o fortalecimento dessas conexões.

Também foram identificados autores mais relevantes na temática com destaque para Blomsma et al (2018) e Daddi et al (2017) sendo estes os autores com publicações mais recentes e com maior número de citações. Acrescente-se que os resultados apontam que o periódico Journal Cleaner Production é, atualmente, o principal destino para esse tipo de publicação.

Quanto a países e publicações, não houve identificação de países destacados quanto à publicações do tema em questão, uma vez que a diferença é considerada pequena e a distribuição quase homogênea. Mas, destaca-se que, embora esse tipo de estudo ainda seja considerado potencial e encontre-se em crescimento, o Brasil apresentou apenas uma das publicações. Como esta é uma área potencial e de crescimento e o Brasil conta com um número significativo de publicações sobre a gestão de pequenas empresas, sugere-se o desenvolvimento de estudos em conexão com a EC. Tal iniciativa poderá fazer o país figurar como um dos núcleos potenciais para pesquisas futuras.

Para estudos futuros, os autores sugerem a realização de estudos de caso na temática, estudos quantitativos em segmentos e ramos de atividades específicos para, possivelmente, fornecer informações mais específicas e detalhadas. Sugere-se, ainda, agregar a simbiose industrial e a economia verde no âmbito dos estudos.

\section{REFERÊNCIAS}


ALI, A. K; WANG Y; ALVARADO J. Facilitating industrial symbiosis to achieve circular economy using value-added by design: A case study in transforming the automobile industry sheet metal waste-flow into Voronoi facade systems, Journal of Cleaner Production, v. 234, n. 10, p.1033-1044, oct 2019

BLOMSMA, F.; BRENNAN, G. The Emergence of Circular Economy - A New Framing Around Prolonging Resource Productivity. Journal of Industrial Ecology, v. 21, n.3, may 2017

BREURE, A.M; LIJZENA, J.P.A; MARINGC, L. Soil and land management in a circular economy. Science of The Total Environment, v.624, n.15, p 1125-1130, may, 2018

CIRCULAR ECONOMY. Sobre a economia circular. 2019. Disponível em https://www.circulareconomy.pt/sobre-economia-circular/ Acesso em 12/11/2019.

CHEN, C. CiteSpace II: Detecting and visualizing emerging trends and transient patterns in scientific literature. InterScience, v.57, n. 3, p. 359-377, 2010.

CRUZ, C; RIBEIRO, U. Metodologia científica: teoria e prática. Rio de Janeiro: Axcel Books, 2003.

ÇOLAK, L.; AKCENGIZ P. Y. Transition from conventional to sustainable production: A case study in OSTIM organized industrial zone. In.: INTERNATIONAL SUSTENTAINABLE BUILDINGS SYMPOSIUM, 3. v 1,p. 525-533, 2018.

DADDI, T.; NUCCI, B.; IRALDO, F.. Using Life Cycle Assessment (LCA) to measure the environmental benefits of industrial symbiosis in an industrial cluster of SMEs. Journal of Cleaner Production, v. 147, p. 157-164, mar,.2017.

DADDI, T., CEGLIA D.; BIANCHI, G.; BARCELlOS. M. D. Paradoxical tensions and corporate sustainability: A focus on circular economy business cases Corporate Social, Responsibility and Environment Management. v. 26, p. 770-780, jul-aug, 2019

DI MAIO, F.; REM, P.C.; BALDÉ, K.; POLDER, M. Measuring resource efficiency and circular economy: A market value approach Resources, Conservation and Recycling, v.. 122, p. 163-171, jul, 2017,

FENG, Y., GUO, Y.; YANG, G.; QIN X; SONG Z. Household biogas development in rural China: On policy support and other macro sustainable conditions. Renewable and Sustainable Energy Reviews, v. 16, e.8, p. 5617-5624, oct, 2012.

GHISELLINI, P. A.; CIALANI, C.; ULGIATI, S.. A review on circular economy: the expected transition to a balanced interplay of environmental and economic systems. Journal of Cleaner Production, v.114, n.7, p.11-32, jan, 2016. 
HAAS, W.; KRAUSMANN, F.; WIEDENHOFER, D.; HEINZ, M. How Circular is the Global Economy?: An Assessment of Material Flows, Waste Production, and Recycling in the European Union and the World in 2005 Journal of Industrial Ecology, v. 19, n.5, p. 765-777, mar, 2015.

LAVILLE, E. A Empresa Verde. e. 1. São Paulo :Editora Õte, 2009.

MACARTHUR, E. Foundation Growth within: a Circular Economy Vision for a Competitive Europe McKinsey Center for Business and Environment, Ellen McArthur Foundation. United Kingdom, 2015. Disponível em <https://www.ellenmacarthurfoundation.org/assets/downloads/publications/EllenMacArthurF oundation Growth-Within July15.pdf> Acesso em: 07 ago. 2019

MULROW, J. S. DERRIBLE S.; ASHTON, W. S. CHOPRA, S. S. Industrial Symbiosis at the Facility Scale .Journal of Industrial Ecology, v.21, n. 3, p.559-571, jun 2017

ORMAZABAL, M., PRIETO-SANDOVAL, V.; PUGA-LEAL, R.; JACA, C.. Circular Economy in Spanish SMEs: Challenges and Opportunities. Journal of Cleaner Production, v. 185 , n.10, mar, 2018

PATRICIO. J., AXELSSON L., BLOMÉ S.; ROSADO, L. Enabling industrial symbiosis collaborations between SMEs from a regional perspective 2018. Journal of Cleaner Production, v.202, n. 20, p. 1120-1130, nov, 2018.

PAULIUK, S., WANG, T., MÜLLER, D.B., Moving toward the circular economy: the role of stocks in the Chinese steel cycle. Environment Science Technology. v.46, n.1, p 148-154,jan., 2012.

PIGOSSO, D., SCHMIEGELOW, A.; ANDERSEN M. M.. Measuring the Readiness of SMEs for Eco-Innovation and Industrial Symbiosis: Development of a Screening Tool. Sustainability, v.10, n.8, aug, 2018

RAZMINIENE, K.,TVARONAVICIENE M. Detecting the linkages between clusters and circular economy 2018. Terra Economicus, Vol 16 N4 2018.

RIZOS, V. et al. Implementation of Circular Economy Business Models by Small and Medium-Sized Enterprises (SMEs): Barriers and Enablers. Sustainability, v. 8., nov, 2016

ROMNÉE, A.; VANDERVAEREN, C.; BREDA, O.; TEMMERMAN, N. A greenhouse that reduces greenhouse effect: how to create a circular activity with construction waste? In: Conference SBE19 BAMB CIRCPATH, Brussels, 2019 
SELLITTO, M. A.; MURAKAMI, F. K. A., Industrial symbiosis: A case study involving a steelmaking, a cement manufacturing, and a zinc smelting plant 2018 Journal Chemical Enginrering Transactions, v, 70, aug 2018.

SINGH, M. ; CHAKRABORTY, A. ; ROY, M. Developing an extended theory of planned behavior model to explore circular economy readiness in manufacturing MSMEs, India. Resources, Conservation and Recycling, v. 135, p. 313-322, aug., 2018

TURJAK, S., UNUKIĆ, I.; LIOVIC D. Aspects of the transition of SMEs in the Republic of Croatia, In.: International May Conference on Strategic Management - IMCSM18, may, 2018, Bor, Serbia, 2018

WEN, Z. and MENG, X. "Quantitative assessment of industrial symbiosis for the promotion of circular economy: a case study of the printed circuit boards industry in China's Suzhou New District”, Journal of Cleaner Production, v. 90, p. 211-219, 2015 